\title{
Associations between immunological function and memory recall in healthy adults
}

Grace Y. Wang ${ }^{1}$, Tamasin Taylor ${ }^{1}$, Alexander Sumich ${ }^{1,}$, Fabrice Merien ${ }^{3}$, Robert Borotkanics $^{4}$, Wendy Wrapson ${ }^{5}$, Chris Krägeloh ${ }^{1}$, Richard J. Siegert ${ }^{1 *}$

${ }^{1}$ Department of Psychology, School of Public Health and Psychosocial Studies, Auckland University of Technology, New Zealand

${ }^{2}$ Division of Psychology, School of Social Sciences, Nottingham Trent University, Nottingham, United Kingdom

${ }^{3}$ AUT-Roche Diagnostics Laboratory, School of Science, Auckland University of Technology, New Zealand

${ }^{4}$ Department of Biostatistics and Epidemiology, School of Public Health and Psychosocial Studies, Auckland University of Technology, New Zealand

${ }^{5}$ National Institute for Public Health and Mental Health Research, School of Public Health and Psychosocial Studies, Auckland University of Technology, New Zealand

Corresponding author: Richard J. Siegert, Department of Psychology, Auckland University of Technology, 90 Akoranga Drive, Auckland 1142, New Zealand; email: richard.siegert@aut.ac.nz

The Funding and Disclosure

The research has been funded by Strategic Research Investment Fund of Auckland University of Technology. The funding source had no role in the collection, analysis, or interpretation of the data or submission. There are no any competing financial interests in relation to the work described. The authors declare no conflict of interest. 


\begin{abstract}
Studies in clinical and aging populations support associations between immunological function, cognition and mood, although these are not always in line with animal models. Moreover, very little is known about the relationship between immunological measures and cognition in healthy young adults. The present study tested associations between the state of immunological functionimmune system and memory recall in a group of relatively healthy adults. Immediate and delayed memory recall was assessed in 30 participants using the computerised cognitive battery. CD4, CD8 and CD69 subpopulations of lymphocytes, Interleukin-6 (IL-6) and cortisol were assessed with blood assays. Correlation analysis showed significant negative relationships between CD4 and the short and long delay memory measures. IL-6 showed a significant positive correlation with long-delay recall. Generalized linear models found associations between differences in all recall challenges and CD4. A multivariate generalized linear model including CD4 and IL-6 exhibited a stronger association. Results highlight the interactions between CD4 and IL-6 in relation to memory function. Further study is necessary to determine the underlying mechanisms of the associations between the state of immune system and cognitive performance.
\end{abstract}

Key words: Immunological measures; memory; T lymphocytes; CD4; Interleukin-6 


\section{Introduction}

A complex network of bidirectional signals exchanged between the nervous, endocrine and immune systems affect cognition and mood (Maier, 2003). For example, chronic stress induces the persistent activation of the hypothalamic-pituitary-adrenal axis and the autonomic nervous system, leading to the release of hormones (e.g. adrenaline, noradrenaline and cortisol) that induce quantitative and qualitative changes in the immune system (Glaser \& Kiecolt-Glaser, 2005). Chronic activation of inflammatory pathways, on-the-other-hand, alters synthesis of certain neurotransmitters (e.g. serotonin), increasing stress responsivity and vulnerability to mood disorders (Kiecolt-Glaser, Derry, \& Fagundes, 2015). Such alterations to neural chemistry would also be expected to affect several cognitive domains, including learning and memory (Švob Štrac, Pivac, \& Mück-Šeler, 2016). Thus, the link between immune system function and cognition is supported in studies of stress (Aas et al., 2014), human immunodeficiency virus (HIV) (Hong \& Banks, 2015), cancer (Andreotti, Root, Ahles, McEwen, \& Compas, 2015), Alzheimer's disease (Raskin, Cummings, Hardy, Schuh, \& A Dean, 2015) and healthy aging (Serre-Miranda et al., 2014). However, the relationship between immunological markers and memory in relatively healthy (nonclinical) young adults remains unclear. Nevertheless, its investigation has important theoretical implications and practical utility with regard to advising on optimum immunological function for psychological wellbeing.

One of the most important components of the adaptive immune response is the lymphocytes, such as $\mathrm{T}$ and B lymphocytes. $\mathrm{T}$ lymphocytes, or $\mathrm{T}$ cells, play a pivotal role in cell-mediated immunity. Primary T cells include helper, killer, regulatory types. T helper cells (TH cells) are involved in the maturation of B cells into antibody producing plasma cells (humoral immunity of the adaptive immune system) and activation of cytotoxic $\mathrm{T}$ cells and 
macrophages. These $\mathrm{TH}$ cells are also known as $\mathrm{CD}^{+} \mathrm{T}$ cells because they express the CD4 glycoprotein on their surfaces. Once activated, TH cells divide rapidly and produce cytokines that regulate the immune response. Killer T cells (e.g. $\left.\mathrm{CD}^{+} \mathrm{T}\right)$ kill tumor cells, cells that are infected (particularly with viruses) and/or damaged cells through complex interactions of molecules between those expressed on the surface of the $\mathrm{T}$ cells and derived from antigenpresenting cells. Multiple surface proteins are known to be upregulated during the immune activation process. CD69 is the earliest inducible surface antigen expressed on lymphocytes after T- or B-cell activation and is absent from resting lymphocytes. Recent evidence supports a regulatory role for CD69 in modulating the inflammatory response (Sancho, Gómez, \& Sánchez-Madrid, 2005).

Cytokines modulate the balance between humoral and cell-based immune responses, and regulate the responsiveness of other cell populations important for brain function (Maier \& Watkins, 2003). For example, cytokines can influence the metabolism of neurotransmitters (e.g. serotonin, norepinephrine and dopamine) and alter neuroendocrine function. That is, acute Interleukin (IL)-2 administration enhances dopaminergic transmission, while systemic IL-6 administration induces increased noradrenergic and serotonergic transmission (Wilson, Finch, \& Cohen, 2002). CD4 ${ }^{+}$T-cells regulate brain-derived neurotrophic factor (BDNF), a protein critical for synaptic plasticity and memory-processing (Lu et al., 2008), and contribute to the neurogenic microenvironment (Wolf et al., 2009). On-the-other-hand, excessive or sustained release of inflammatory cytokines produces neurotoxic effects on neurons and glial cells, at least in vitro (Allan \& Rothwell, 2001; Hanisch, 2002).

Animal studies support the importance of CD4+ T cells in hippocampal neurogenesis and memory (Jeon et al., 2016). However, levels of $\mathrm{CD}^{+}$cells have been inversely associated with memory recallimmediate and stored memory in children infected with HIV (RuisenorEscudero et al., 2016) and short-term verbal memory, verbal working memory in older adults 
(Serre-Miranda et al., 2015). Other cells were also inversely associated with stored memory, including $\mathrm{CD}_{38}{ }^{+}$and CD8+ (Ruisenor-Escudero et al., 2016). CD69 is also implicated in memory and has been found on monocytes derived from patients with Alzheimer's disease (Kusdra, Rempel, Yaffe, \& Pulliam, 2000). In women with major unipolar depression, which is associated with increases in IL-6, verbal memory performance is negatively associated with IL-6 plasma level after controlling for age, years of education and depression severity (GrassiOliveira, Bauer, Pezzi, Teixeira, \& Brietzke, 2011). Consistently, an aging population study reported that people with high IL-6 level exhibited poorer attention and sensory memory compared to those having a low level or normal levels of IL-6 (Elwan et al., 2003). These findings provide initial evidence for the relationship between biomarkers of the state of immune function-system and cognitive function in aging and clinical populations. However, as mentioned, little is known about the relationship between the immune system and cognition in the general young adult population.

The present study was designed to examine whether associations between a set of immunological measures and memory function observed in clinical populations are consistent in healthy populations. Three levels of memory recall were evaluated, including immediate,

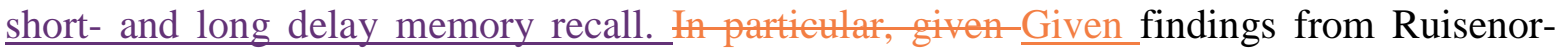
Escudero et al. (2016) and Kusdra et al. (2000) we expected an inverse associations between memory recall and both $\mathrm{CD}^{+}$and CD8+. Given those of Grassi-Oliveira et al. (2011), we anticipated an inverse association between memory recall and IL-6. In addition, associations between memory and other measures of the state of immune systemfunction (cortisol, CD69) were explored.

\section{Materials and Methods}




\subsection{Participants}

Participants (n=30; 10 males, 20 females, $M_{\text {age }}=29.50$ years, $S D_{\text {age }}=11.97 ; M_{\text {education }}=15.50$ years, $\left.S D_{\text {education }}=2.5\right)$ included undergraduate $(n=25)$, post-graduate $(n=4)$ students and staff $(n=1)$ from a university in Auckland, New Zealand who enrolled in a large study investigating the effect of mindfulness on brain function. All data currently reported were collected prior to their engagement in mindfulness practice and outside of the school exam period, to avoid any possible influence of exam stress on immune system functioning. Exclusion criteria included current severe depression, a history of serious brain injury, epilepsy, drug or alcohol problem, and current prescription of medication for psychiatric illness (e.g. anxiety, depression, schizophrenia). Current depression level was measured using the Depression subscale from the Depression, Anxiety and Stress scale (DASS; (Benjamini \& Hochberg, 1995)) and the total score over 20 suggested severe or extremely severe depression. Students of members of the research team were also excluded.

The ethnic makeup included, NZ European $(n=16)$, Indian $(n=3)$, Asian $(n=3)$, Māori $(n$ $=1)$, Pacific $(n=1)$, others $(n=4)$ and Not Specified $(n=2)$. Ethical approval was granted by Auckland University of Technology Ethics Committee, and written informed consent was given by all participants. Students were gifted a $\$ 20$ voucher for their time contributed to the study.

\subsection{Memory recall and Recognition test}

Testing for immediate, short and long delay memory recall was taken during the normal office hour-of a day $\mathrm{s}(9 \mathrm{am}-5 \mathrm{pm})$. Immediate and delayed memory recall was assessed using an Internet-based cognitive battery 'IntegNeuro' (for details see www.brainresource.com). The 'IntegNeuro' test battery has good validity and test-retest reliability (Kemp et al., 2005; Paul et al., 2005; Williams et al., 2010) and meets standardised norms pre-established in 1000 
healthy participants (Clark et al., 2006). It has also been validated against traditional paperand-pencil neuropsychological tests of cognition (convergent validity measures $>0.53$ ) (Paul et al., 2005). This test battery was delivered using an IBM screen interfaced platform with standardised vocal and visual instructions. Instructions were delivered via stereo headphones/microphone at the beginning of each test. Participants were also given a practice set for each test to ensure they understood the instructions.

In the first part, the participants were presented with a list of 12 words, which they were asked to memorise. The list was presented 4 times in total, and the participants were required to recall as many words as possible after each presentation. Immediate recall was measured as the average of the number of words recalled immediately after each presentation.

The participants were then presented with a second list of 12 distracter words, in which none of the words were phonetically or semantically related to the first list, and asked to recall that second list. After this distraction, the participants were asked immediately to recall the previously presented words from the original list. Short delay recall was measured as the number of words recalled from the original list. Long delay recall was measured as the number of words recalled from the original list after a delay of approximately 25 minutes, during which time participants completed other cognitive tests.

\subsection{Blood samples}

Blood samples were taken between 10 am and 12:30 pm on a day different from that on which cognitive tests were performed. This was due to logistic reasons, to avoid the effects of cognitive test on immune response and minimise diurinal variation of circulating Interleukin6 in humans (Nilsonne, Lekander, Åkerstedt, Axelsson, \& Ingre, 2016). Also, cortisol levels were expected to be on the decline at this time (10:00-12:30), after the early morning peak (Levine, Zagoory-Sharon, Feldman, Lewis, \& Weller, 2007). Nevertheless, evidence shows 
that there is considerable consistency on immune responses to acute laboratory stressors induced by cognitive tasks across time and across similar tasks (Adler et al., 2002; Cohen \& Hamrick, 2003).

Blood samples were collected via venepuncture in K2EDTA tubes and tubes with gel SST for serum separation (Surgical Supplies Ltd., Auckland, New Zealand). The EDTA samples were immediately analysed for red and white blood cell counts, haematocrit and haemoglobin concentration on a Sysmex XT automated quantitative haematology analyser (Sysmex Corporation, Auckland, New Zealand). Tubes with separating gels were centrifuged at $4{ }^{\circ} \mathrm{C}$ and $3000 \mathrm{rpm}$ for $15 \mathrm{~min}$. Serum was then extracted, and samples were processed immediately or kept frozen at $-20^{\circ} \mathrm{C}$ until later analysis.

CD4, CD8 and CD69 subpopulations of lymphocytes were assessed with a Muse Cell Analyser (Merck Millipore, USA). The Muse Cell Analyser features miniaturised fluorescence detection and microcapillary technology for rapid, accurate and reliable quantitative cell analysis. Briefly, the Muse ${ }^{\mathrm{TM}}$ Human CD4 T Cell Kit allows for the detection and identification of lymphocytes and CD4 T lymphocytes using a specific CD4 antibody that identifies human helper/inducer CD4+ T cell (HLA Class II reactive) and recognizes a 60kDa surface antigen. The Muse Human CD8 T Cell Kit allows for the detection and identification of lymphocytes and CD8 T lymphocytes using a specific CD8 antibody that identifies a 68-kDa transmembrane glycoprotein (HLA Class I restricted). Finally, the Muse Human Lymphocyte CD69 Assay uses an anti-lymphocyte cocktail that identifies the total lymphocyte population and a specific antibody that binds to the CD69 early activation marker expressed on lymphocytes. T-cell concentration in cells/ $\mu \mathrm{L}, \mathrm{T}$-cell percent of lymphocytes and total lymphocyte concentration in cells $/ \mu \mathrm{L}$ were obtained for all subpopulations tested. All kits were used according to manufacturer's instructions for the processing of whole blood. 
Cortisol and Interleukin-6 (IL-6) concentrations were calculated using specific assays on a cobas Modular E170 analyser (Roche Diagnostics, New Zealand). Total duration of assay was $18 \mathrm{~min}$ based on the electrochemiluminescence principle using ruthenium-conjugated monoclonal antibodies. C-reactive protein (CRP) was measured on a cobas Modular P800 (Roche Diagnostics, New Zealand) based on a Particle-enhanced immunoturbidimetric principle using latex particles coated with monoclonal anti-CRP antibodies. Quantitative results were determined via instrument-specific full point calibration curves for all assays. The detection limit was $1.5 \mathrm{pg} / \mathrm{ml}$ for IL-6.

\subsection{Statistical analyses}

Data were inspected for normality, and where data showed non-normality, log transformations were applied. Spearman's Rho correlations were used to test associations between the memory measures and immunological markers given the skewness of the dataset. Immunological $\underline{\text { (CD4, CD8, CD69, CRP and IL-6) and memory variables (immediate, short- and long delay }}$ recall scores) were included in the statistical analysis. Due to multiple comparisons, alpha criteria were set using Benjamin and Hochberg's (Benjamini \& Hochberg, 1995) step-up procedure. Univariate generalized linear models, followed by multivariate models were developed, including jackknife analyses. Final models were selected that exhibited the lowest Bayesian Information Criteria (BIC). The statistical procedures were performed using Stata version 14.

\section{Results}

Table 1 shows the descriptive statistics for the sample as well as the memory scores and results from blood assay analysis. All of the memory scores were found to be significant with CD4 
and IL-6, however, aAfter controlling for multiple comparisons using Benjamin Hochberg step-up procedure, the significant association between immediate recall and CD4 and between short delay recall and IL-6 disappeared. correlations between immunological, and memory variables revealed that $\mathrm{On}$ the other hand, $\mathrm{CD} 4$ showed significant negative relationships with short delay recall $(r=-.53, p=.03)$ and long delay recall $(r=-.54, p=.02)$. IL-6 showed a significant positive correlation with long delay recall $(r=.46, p=.04)$. None of the memory scores were significantly correlated with CD8, CD69, CRP, or cortisol (Table 2 and Figure 1 $\underline{\text { show these relationships) }}$

Tables 3, 4 and 5 show the results of the generalized linear models for immediate, short delay and long delay recall respectively. CD4 was significantly inversely associated with immediate, short delay and long delay. In addition, for short delay, significant associations were observed for IL-6 (positive) and CRP (inverse). Model 2 which included these parameters had a significantly better fit than the model with CD4 alone $\left(X^{2}=14.00, \mathrm{df}=2, p\right.$ $=.007)$. Likewise, for long term delay, the model that included both CD4 and IL-6 had a better fit than that with $\mathrm{CD} 4$ alone $\left(X^{2}=5.18, \mathrm{df}=1, p=.023\right)$.

\section{Discussion}

Evidence suggests that direct immune processes are controlled by changes in neural activity and, concomitantly, immune-derived induction of cytokines can interfere with brain function (Maier \& Watkins, 2003). The current study investigated whether associations between immunological markers and memory recall observed in clinical populations are present in the general population. Results suggest memory recall is associated with CD4, IL-6 and CRP.

The inverse association between CD4 and recall contrasts studies in animals, which generally suggests CD4 T cells play a supportive role in brain function, including hippocampal function, 
neurogenesis and memory. For example, decreased brain-derived neurotrophic factor (BDNF) production has been found in the brains of the CD4-depleted mice (Wolf et al., 2009). However, the current results are in line with previous human studies, for example in children with HIV infection (Ruisenor-Escudero et al., 2016). Also, in a study in healthy aging, better performance in memory and executive function were found for those with lower bloodderived CD4 T-cell counts and higher B-cell counts (Serre-Miranda et al., 2015). In that study, it was proposed that high CD4 $\mathrm{T}$ cells reflect a more proinflammatory profile, which could underpin poorer memory. In agreement with this, short delay recall was also inversely associated with CRP, a nonspecific marker of inflammation.

IL_6 was positively associated with long delay recall and strengthened the association of CD4 with long delay recall. This is in line with animal studies which have shown that IL-6 increases serotoninergic and dopaminergic transmission in the hippocampus and promotes the survival of catacholaminergic neurons (Zalcman et al., 1994). Nonetheless, an inverse relationship between IL-6 and memory has been observed in clinical populations, such as people with dementia and major depression (Elwan et al., 2003; Grassi-Oliveira et al., 2011). Taken together, the presence of depression in clinical populations may mediate the relationships between immunological measures and cognition. IL-6 is typically raised in these populations, and it is possible that the role of IL-6 in memory varies based on the conditions or context in which it is elevated, as well as on the magnitude and duration of elevation. In support of this idea, memory function of patients with chronic fatigue syndrome or control subjects were unaffected by administration of IL-6 (Arnold et al., 2002), and surgical patients with elevated IL-6 levels exhibited less declines in declarative memory after surgery (Shapira-Lichter et al., 2008). For systemic lupus erythematosus patients, those with higher levels of IL-6 exhibited higher learning scores (Kozora, Laudenslager, Lemieux, \& West, 2001). An inverted U-shape relationship between IL-6 and cognitive function has been proposed, suggesting that cognition 
may be facilitated by moderate levels of IL-6 but adversely affected when IL-6 levels are either very low or very high (Kozora et al., 2001). In our study, we only included healthy volunteers whose IL-6 levels are more likely to be moderate, and this may explain the observed positive association between IL-6 and memory. Furthermore, it should be noted that the involvement of IL-6 was only observed in delay recall, not in immediate recall, which suggestsand this may be due to the involvement of there are-separate neural circuits or memory store support in the short term and long term memory (Ranganath \& Blumenfeld, 2005).

No strong correlations were observed between memory and either CD8, CD69 or cortisol, suggesting that none of these immunological markers are tightly linked to memory in our study group. The reasons for the absence of correlations between memory and these immunological measures are unclear and need to be further investigated in larger groups. Nevertheless, it has been shown that $\mathrm{CD} 8^{+} \mathrm{T}$ cell compartment remains functionally intact in mice exhibiting significant impaired performance in memory tasks (Radjavi, Smirnov, \& Kipnis, 2014). The association between cortisol and memory is only observed in males but not in females (Wolf, Schommer, Hellhammer, McEwen, \& Kirschbaum, 2001).

As a limitation, the observational design of the study does not allow us to conclude that the link between immunological variables and memory performance represents a causal or timerelated relationship. Furthermore, there was unbalanced gender composition in our study sample, and there could be additional variables that we have not measured that affect both memory and the state of immune function-system and that require further investigation, including gender, body mass index, smoking status or sickness during the test. Nevertheless, our findings support the further exploration of immunological markers and their possible associations and prediction of memory performance as warranted. Future studies should investigate a causal relationship by testing whether manipulation of CD4 and IL1-6 cell counts 
affects memory. Eligibility for this study included healthy/non- clinical population. People with acute mental health conditions on medication (e.g. anxiety disorders, major depression, schizophrenia), and those with a history of traumatic brain injury, alcohol or substance abuse, epilepsy and psychotic disorder were excluded. The findings derived from a sample of 30, mainly young university students and may have limited generalisability to less educated, older or younger groups. Future research should compare across a range of demographics in clinical and non-clinical populations. Blood samples and memory evaluation were performed on $\underline{\text { separate days in order to minmise the effect of individual difference in response to task-related }}$ stress on the state of immune system. Nevertheless, it is important to characterize immune $\underline{\text { responses to acute task-related stress over a longer period time, such as magnitude of response, }}$

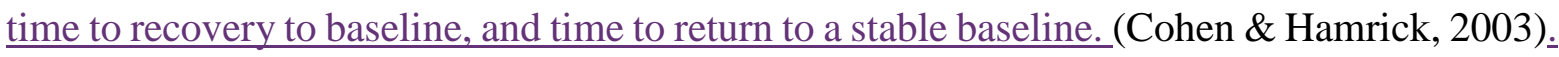
In summary, the current findings suggest that there are associations between immunological markers and memory. Early evaluation and intervention for cognitive problems may benefit from addressing the variations of these immunological makers. Further study is necessary to determine the underlying mechanisms of the associations between immune system and cognitive performance. 


\section{Acknowledgements}

The authors would like to thank David Anstiss and Oleg Medvedev for their help during data collection. 


\section{References}

Aas, M., Dazzan, P., Mondelli, V., Melle, I., Murray, R. M., \& Pariante, C. M. (2014). A systematic review of cognitive function in first-episode psychosis, including a discussion on childhood trauma, stress, and inflammation. Frontiers in psychiatry, 4, 182.

Adler, K. A., Mills, P. J., Dimsdale, J. E., Ziegler, M. G., Patterson, T. L., Sloan, R. P., \& Grant, I. (2002). Temporal Stability of Acute Stress-Induced Changes in Leukocyte Subsets and Cellular Adhesion Molecules in Older Adults. Brain, Behavior, and Immunity, 16(3), 262274.

Allan, S. M., \& Rothwell, N. J. (2001). Cytokines and acute neurodegeneration. Nature Reviews Neuroscience, 2(10), 734-744.

Andreotti, C., Root, J. C., Ahles, T. A., McEwen, B. S., \& Compas, B. E. (2015). Cancer, coping, and cognition: a model for the role of stress reactivity in cancer - related cognitive decline. Psycho - Oncology, 24(6), 617-623.

Arnold, M. C., Papanicolaou, D. A., O'Grady, J. A., Lotsikas, A., Dale, J. K., Straus, S. E., \& Grafman, J. (2002). Using an interleukin-6 challenge to evaluate neuropsychological performance in chronic fatigue syndrome. Psychol Med, 32(6), 1075-1089.

Benjamini, Y., \& Hochberg, Y. (1995). Controlling the false discovery rate: a practical and powerful approach to multiple testing. Journal of the royal statistical society. Series $B$ (Methodological), 289-300.

Clark, C. R., Paul, R. H., Williams, L. M., Arns, M., Fallahpour, K., Handmer, C., \& Gordon, E. (2006). Standardized assessment of cognitive functioning during development and aging using an automated touchscreen battery. Archives of Clinical Neuropsychology, 21(5), 449467.

Cohen, S., \& Hamrick, N. (2003). Stable individual differences in physiological response to stressors: Implications for stress-elicited changes in immune related health. Brain, behavior, and immunity, 17(6), 407-414.

Elwan, O., Madkour, O., Elwan, F., Mostafa, M., Abbas Helmy, A., Abdel-Naseer, M., Abdel Shafy, S., \& El Faiuomy, N. (2003). Brain aging in normal Egyptians: cognition, education, personality, genetic and immunological study. Journal of the Neurological Sciences, 211(12), 15-22.

Glaser, R., \& Kiecolt-Glaser, J. K. (2005). Stress-induced immune dysfunction: implications for health. [10.1038/nri1571]. Nat Rev Immunol, 5(3), 243-251.

Grassi-Oliveira, R., Bauer, M. E., Pezzi, J. C., Teixeira, A. L., \& Brietzke, E. (2011). Interleukin-6 and verbal memory in recurrent major depressive disorder. Neuro Endocrinol Lett, 32(4), 540-544.

Hanisch, U. K. (2002). Microglia as a source and target of cytokines. Glia, 40(2), 140-155.

Hong, S., \& Banks, W. A. (2015). Role of the immune system in HIV-associated neuroinflammation and neurocognitive implications. Brain, behavior, and immunity, 45, 112.

Jeon, S. G., Kim, K. A., Chung, H., Choi, J., Song, E. J., Han, S.-Y., Oh, M. S., Park, J. H., Kim, J.-i., \& Moon, M. (2016). Impaired memory in OT-II transgenic mice is associated with decreased adult hippocampal neurogenesis possibly induced by alteration in Th2 cytokine levels. Molecules and Cells, 39(8), 603.

Kemp, A. H., Cooper, N., Hermens, G., Gordon, E., Bryant, R. A., \& Williams, L. M. (2005). Toward an integrated profile of emotional intelligence: introducing a brief measure. J. Integr. Neurosci, 4, 41-61.

Kiecolt-Glaser, J. K., Derry, H. M., \& Fagundes, C. P. (2015). Inflammation: depression fans the flames and feasts on the heat. American journal of psychiatry, 172(11), 1075-1091. 
Kozora, E., Laudenslager, M., Lemieux, A., \& West, S. G. (2001). Inflammatory and hormonal measures predict neuropsychological functioning in systemic lupus erythematosus and rheumatoid arthritis patients. J Int Neuropsychol Soc, 7(6), 745-754.

Kusdra, L., Rempel, H., Yaffe, K., \& Pulliam, L. (2000). Elevation of CD69+Monocyte/Macrophages in Patients with Alzheimer's Disease. Immunobiology, 202(1), 26-33.

Levine, A., Zagoory-Sharon, O., Feldman, R., Lewis, J. G., \& Weller, A. (2007). Measuring cortisol in human psychobiological studies. Physiol Behav, 90(1), 43-53.

Maier, S. F. (2003). Bi-directional immune-brain communication: Implications for understanding stress, pain, and cognition. Brain, Behavior, and Immunity, 17(2), 69-85.

Maier, S. F., \& Watkins, L. R. (2003). Immune-to-central nervous system communication and its role in modulating pain and cognition: Implications for cancer and cancer treatment. Brain, Behavior, and Immunity, 17(1, Supplement), 125-131.

Nilsonne, G., Lekander, M., Åkerstedt, T., Axelsson, J., \& Ingre, M. (2016). Diurnal Variation of Circulating Interleukin-6 in Humans: A Meta-Analysis. PLOS ONE, 11(11), e0165799.

Paul, R., Lawrence, J., Williams, L., Richard, C., Cooper, N., \& Gordon, E. (2005). Preliminary validity of 'IntegNeuro': A new computerized battery of neurocognitive tests International Journal of Neuroscience, 115, 1549-1567.

Radjavi, A., Smirnov, I., \& Kipnis, J. (2014). Brain antigen-reactive CD4+ T cells are sufficient to support learning behavior in mice with limited T cell repertoire. Brain, behavior, and immunity, 35, 58-63.

Ranganath, C., \& Blumenfeld, R. S. (2005). Doubts about double dissociations between shortand long-term memory. Trends in cognitive sciences, 9(8), 374-380.

Raskin, J., Cummings, J., Hardy, J., Schuh, K., \& A Dean, R. (2015). Neurobiology of Alzheimer's disease: integrated molecular, physiological, anatomical, biomarker, and cognitive dimensions. Current Alzheimer Research, 12(8), 712-722.

Ruisenor-Escudero, H., Familiar-Lopez, I., Sikorskii, A., Jambulingam, N., Nakasujja, N., Opoka, R., Bass, J., \& Boivin, M. (2016). Nutritional and Immunological Correlates of Memory and Neurocognitive Development Among HIV-Infected Children Living in Kayunga, Uganda. J Acquir Immune Defic Syndr, 71(5), 522-529.

Sancho, D., Gómez, M., \& Sánchez-Madrid, F. (2005). CD69 is an immunoregulatory molecule induced following activation. Trends in immunology, 26(3), 136-140.

Serre-Miranda, C., Roque, S., Santos, N. C., Portugal-Nunes, C., Costa, P., Palha, J. A., Sousa, N., \& Correia-Neves, M. (2015). Effector memory CD4+ T cells are associated with cognitive performance in a senior population. Neurology-Neuroimmunology Neuroinflammation, 2(1), e54.

Shapira-Lichter, I., Beilin, B., Ofek, K., Bessler, H., Gruberger, M., Shavit, Y., Seror, D., Grinevich, G., Posner, E., Reichenberg, A., Soreq, H., \& Yirmiya, R. (2008). Cytokines and cholinergic signals co-modulate surgical stress-induced changes in mood and memory. Brain Behav Immun, 22(3), 388-398.

Švob Štrac, D., Pivac, N., \& Mück-Šeler, D. (2016). The serotonergic system and cognitive function. Translational Neuroscience, 7(1), 35-49.

Williams, L. M., Hermens, D. F., Thein, T., Clark, C. R., Cooper, N. J., Clarke, S. D., Lamb, C., Gordon, E., \& Kohn, M. R. (2010). Using Brain-Based Cognitive Measures to Support Clinical Decisions in ADHD. Pediatric Neurology 42(2), 118-126.

Wilson, C. J., Finch, C. E., \& Cohen, H. J. (2002). Cytokines and Cognition-The Case for A Head-to-Toe Inflammatory Paradigm. Journal of the American Geriatrics Society, 50(12), 2041-2056. 
Wolf, O. T., Schommer, N. C., Hellhammer, D. H., McEwen, B. S., \& Kirschbaum, C. (2001). The relationship between stress induced cortisol levels and memory differs between men and women. Psychoneuroendocrinology, 26(7), 711-720.

Wolf, S. A., Steiner, B., Akpinarli, A., Kammertoens, T., Nassenstein, C., Braun, A., Blankenstein, T., \& Kempermann, G. (2009). CD4-Positive T Lymphocytes Provide a Neuroimmunological Link in the Control of Adult Hippocampal Neurogenesis. The Journal of Immunology, 182(7), 3979-3984.

Zalcman, S., Green-Johnson, J. M., Murray, L., Nance, D. M., Dyck, D., Anisman, H., \& Greenberg, A. H. (1994). Cytokine-specific central monoamine alterations induced by interleukin-1,-2 and-6. Brain research, 643(1), 40-49. 
Table 1. Medians, interquartile ranges of demographics, immunological and memory measures $(N=30)$.

\begin{tabular}{|c|c|c|}
\hline & Median & $\begin{array}{l}\text { Interquartile } \\
\text { range }\end{array}$ \\
\hline Age (years) & 25 & 14 \\
\hline Education (years) & 15.50 & 2.0 \\
\hline CD4 (cells/ $\mu \mathrm{L})$ & 812.50 & 268.5 \\
\hline CD8 (cells/ $\mu \mathrm{L})$ & 493.50 & 259.5 \\
\hline CD69 (cells $/ \mu \mathrm{L})$ & 14.00 & 14.3 \\
\hline IL-6 (pg/ml) & 4.39 & 2.58 \\
\hline $\mathrm{CRP}(\mathrm{mg} / \mathrm{L})^{1}$ & 1.00 & 3.00 \\
\hline Cortisol (nmol/L) & 272.05 & 271.2 \\
\hline Immediate recall $^{2}$ & 33.50 & 5 \\
\hline Short delay recall ${ }^{2}$ & 8.50 & 2 \\
\hline Long delay recall ${ }^{2}$ & 8.00 & 3 \\
\hline
\end{tabular}

${ }^{1}$ C-Reactive Protein, ${ }^{2}$ Measured by number of words recalled. 
Table 2. Correlations between immunological and memory measures ${ }^{1}$.

\begin{tabular}{llllllllll}
\hline & 1 & 2 & 3 & 4 & 5 & 6 & 7 & 8 & 9 \\
\hline 1.CD4 & - & & & & & & & & \\
2.CD8 & .22 & - & & & & & & & \\
3.CD69 & -.07 & .10 & - & & & & & & \\
4.IL-6 & -.34 & .01 & -.20 & - & & & & & \\
5.C- Reactive Protein & -.23 & .18 & .03 & .43 & - & & & & \\
6.Cortisol & -.07 & .05 & -.00 & -.37 & .13 & - & & \\
7.Immediate recall & -.45 & .13 & -.01 & .16 & -.19 & .21 & - & & \\
8.Short delay recall & $-.53^{* *}$ & .02 & .00 & .44 & -.04 & -.25 & $.58^{* *}$ & - & \\
9.Long delay recall & $-.54^{* *}$ & -.04 & .21 & $.46^{*}$ & .07 & -.25 & .41 & $.70^{* *}$ & -
\end{tabular}

${ }^{1} \mathrm{P}$-values corrected for multiple comparisons (consider significant $<0.01$ after correction). ${ }^{* *}$ Correlation is significant level (2-tailed). 


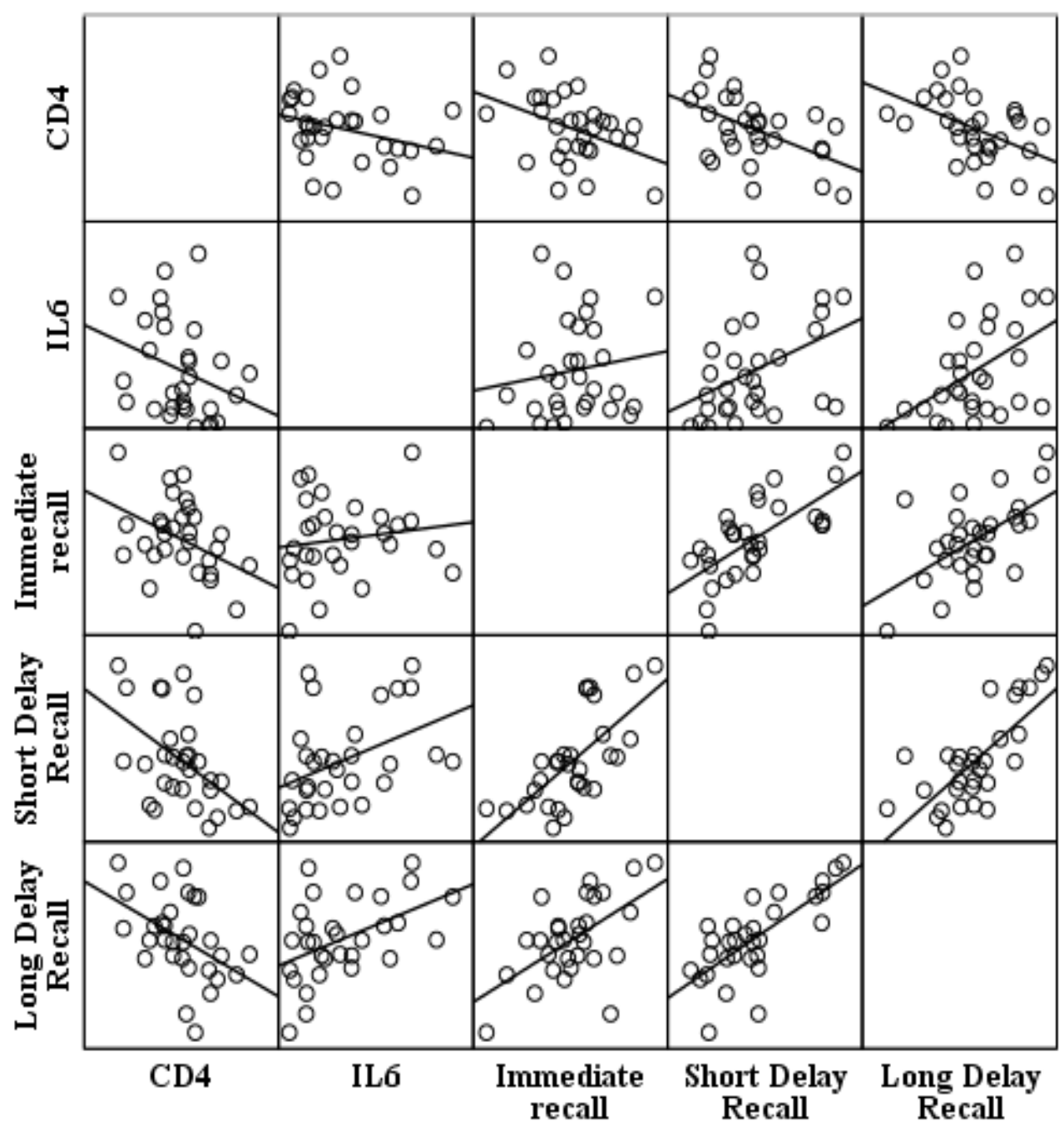

Figure 1. Scatterplot Matrix depicting correlations between memory scores and immune function measures. 
Table 3. Generalized linear model of total immediate recall trials $(N=30)$.

\begin{tabular}{lllllll}
\hline Variable & Exp(b) & $\begin{array}{l}\text { SE } \\
\text { (Jackknife) }\end{array}$ & & T & P & CI \\
& & & & & \\
\hline CD4 & 0.898 & 0.004 & 2.77 & $<0.001$ & 0.982 & - \\
& & & & & 0.997 & \\
Constant & $1.05^{18}$ & $3.38^{18}$ & 12.84 & $<0.001$ & $1.41^{15}$ & - \\
& & & & & $7.76^{20}$ & \\
\hline Overall model fit: & & AIC & 17.634 & BIC & 17.634
\end{tabular}


Table 4. Generalized linear models of short delay recall.

Model 1 Summary:

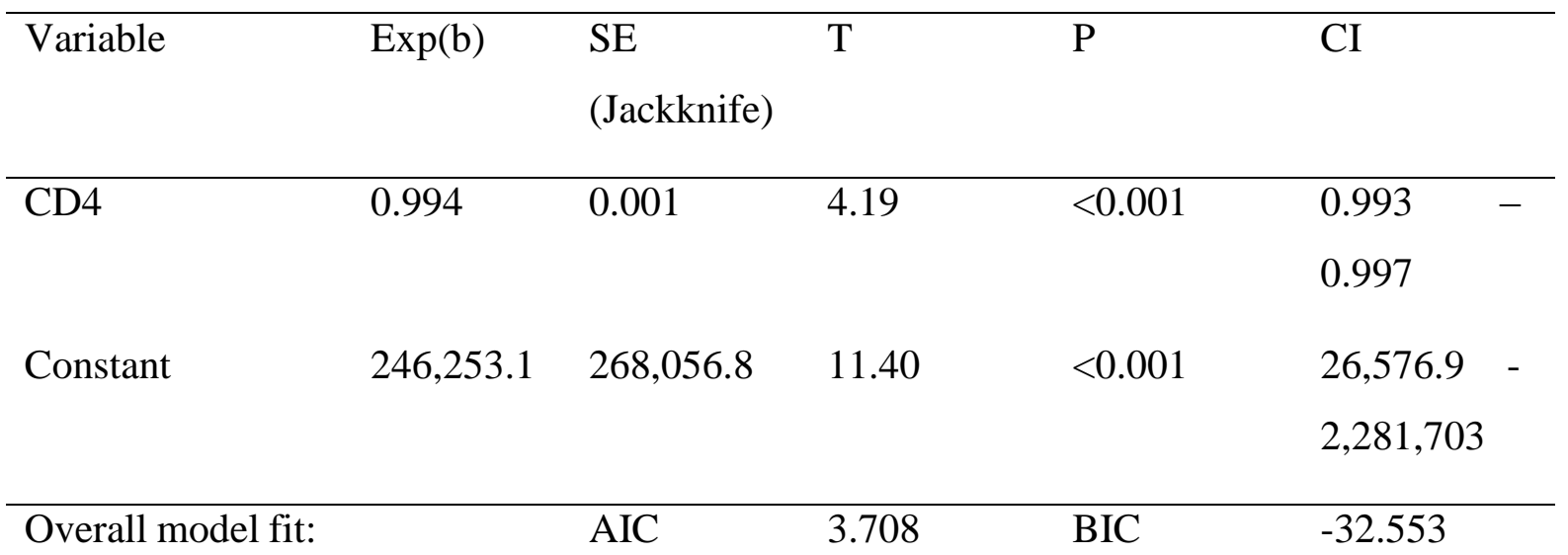

Model 2 Summary:

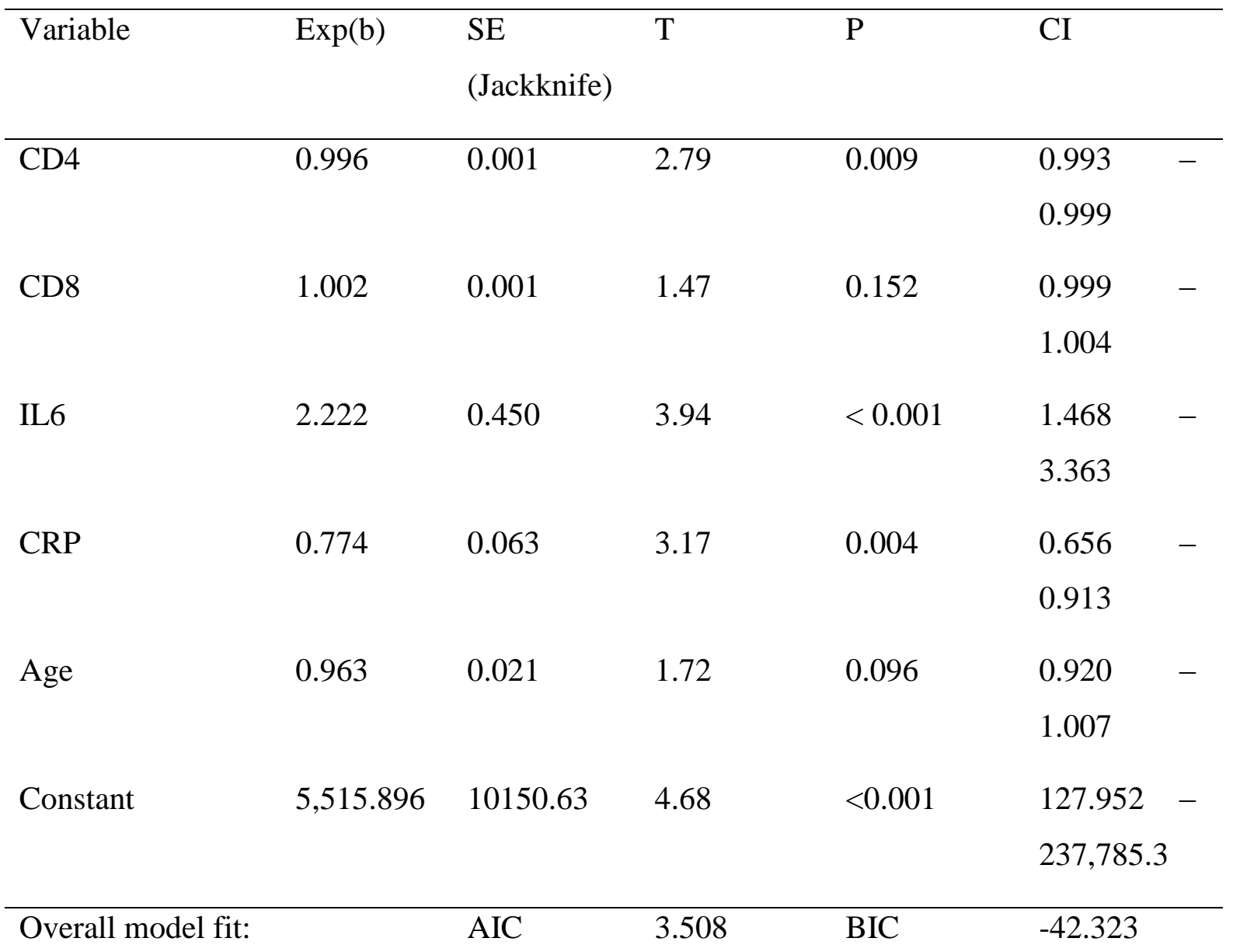


Table 5. Generalized linear models of long delay recall.

Model 1 summary:

\begin{tabular}{llllll}
\hline Variable & Exp(b) & $\begin{array}{l}\text { SE } \\
\text { (Jackknife) }\end{array}$ & T & P & CI \\
& & & & \\
\hline CD4 & 0.994 & 0.001 & 4.97 & $<0.001$ & 0.991 \\
& & & & & 0.997 \\
& & & & & $32,943.02-$ \\
Constant & $247,267.8$ & $243,696.8$ & 12.60 & $<0.001$ & $1,855,973$ \\
& & & & & 35.707
\end{tabular}

Model 2 Summary:

\begin{tabular}{llllll}
\hline Variable & Exp(b) & $\begin{array}{l}\text { SE } \\
\text { (Jackknife })\end{array}$ & T & P & CI \\
& & & & \\
\hline CD4 & 0.995 & 0.001 & 4.28 & $<0.001$ & $0.993-0.997$ \\
IL6 & 1.757 & 0.498 & 1.99 & 0.057 & $0.983-3.139$ \\
Constant & $5,909$. & $11,819.33$ & 4.34 & $<0.001$ & 98.843 \\
& 252 & & & & $353,280.8$ \\
\hline Overall model fit: & & AIC & 4.339 & BIC & 18.350
\end{tabular}

